\title{
From Near-Synchronously Rotating Planets to Tidal Lock: A New Class of Habitable Planets Examined for Forest Habitability
}

\author{
Martin J. Heath \\ 47 Tulsemere Road, West Norwood, London SE27 9EH, UK.
}

\author{
Laurance R. Doyle
}

SETI Institute, 2035 landings Drive, Mountain View, CA 94043, USA.

\begin{abstract}
Haberle et al. (1996) and Joshi, Haberle \& Reynolds (1997) demonstrated that for a planet receiving Earth-level insolation, a $100 \mathrm{mb}$ pure $\mathrm{CO}_{2}$ atmosphere would carry enough heat flux to the dark side of a synchronously rotating planet (SRP) to prevent the atmosphere freezing out there. For a $1,500 \mathrm{mb}$ pure $\mathrm{CO}_{2}$ atmosphere and 0.8 Earth insolation, liquid water could survive over much of the planet. Such high partial pressures of $\mathrm{CO}_{2}$ may seem like special pleading, but in fact, they are not essential for habitable conditions. The effective grey optical depth of the present terrestrial atmosphere (with just $350 \mathrm{ppm} \mathrm{CO}_{2}$, and $\mathrm{H}_{2} \mathrm{O}$ as the principal greenhouse gas) is approximately 0.9 , as against 1.0 for a 1,000 mb pure $\mathrm{CO}_{2}$ atmosphere, so the latter serves merely as a useful approximation (for a truly Earth-type atmosphere, temperatures would be a few degrees lower over the lit hemisphere). Heath et al. (1999) concluded that even forest-habitable conditions, suitable for Earth-like trees (Heath 1996) were not to be ruled out on the basis of present knowledge. Recent work by Joshi (2004) has modelled the range of climatic options for certain land-sea distributions, and these leave the door open for further investigations of combinations of geography and insolation compatible with forest-habitability.
\end{abstract}

Kasting et al. (1993) took the orbital radius at which tidal lock would be complete by $4.5 \mathrm{Gyr}$ as delineating a margin of the habitable zone. Obviously, planets which achieved synchronous rotation at Earth age, must have spent the rest of their careers rotating relative to their parent stars. They might start as fast rotators, slow through a regime in which rotation failed to stablise them against chaotic obliquity, and then achieve stable obliquity as they slow even further.

We identify here a new class of habitable planet whose existence has been hitherto unrecognised. This is the class of near-synchronously rotating planets (NSRP), which will offer a distinctive regime, posing severe problems for the survival of higher plants assuming, for the purpose of modelling, that such plants exhibit similar physiological tolerances to those on Earth.

The length of a planet's day-night cycle will depend on the rate of rotation of a planet, and on the angular displacement of the Sun in the sky resulting 
from the planet's orbital motion. In the case of the Earth, the orbital motion completed in one day is sufficient to add, on the average about 4 minutes to the day. The Earth's sidereal period, the time taken to rotate once with regard to the stars, is some 23 hours 56 minutes (note that the Earth is not in a perfectly circular orbit, so the magnitude of the effect varies throughout the year). Severe discrepancies between rotation rate and the day-night cycle will appear when the rotation rate and orbital period differ only very slightly.

The length of a planet's day-night cycle may be calculated from the simple relation:

$$
\Theta=\frac{P_{\text {orb }}}{\left(P_{\text {orb }} / P_{\text {rot }}-1\right)}
$$

where $\Theta$ is the length of the day-night cycle, $P_{\text {orb }}$ is the planet's orbital period, and $P_{\text {rot }}$ is the planet's sidreal rotation period (which would be negative for initial retrograde rotation).

One can examine the length of a planet's day-night cycle (expressed in terms of a planet's orbital period) against the rate of the planet's sidereal period of rotation (expressed in terms of the rate corresponding to synchronous lock). Circular orbits will herein be assumed. It is evident that there are two possible (and symmetrical) profiles of spin down, with $\Theta=\infty$ when the planet's spin rate matches the orbital period. One profile represents spin-down with the sun circling prograde across the planet's skies, until synchronous lock is achieved. The other profile represents spin-down with the sun moving retrograde across the planet's skies. The two situations are not equivalent, despite the profiles' symmetry, and there are important differences between them. Three rotational domains may be distinguished.

Domain I represents the spin-down of a planet from a state of prograde (direct) rotation into tidal lock. At no time during tidal spin-down does sidereal rotation cease, or become retrograde. The apparent motion of the sun in the sky is always prograde. Domain III represents the early spin-down phase of a planet whose sidereal rotation is retrograde. The motion of the sun across the sky is retrograde. A planet spinning retrograde must be slowed to zero sidereal rotation (with interesting implications for meteorology and geophysics) before being spun up to the rate of prograde rotation corresponding to synchronous lock. Domain II includes all prograde rotation rates between zero and synchronous lock. The apparent motion of the sun in the sky would be retrograde.

For a planet spinning progressively slower under influence of a stellar tide, the day-night cycle will become steadily longer. Initially, the day-night cycle will correspond, to a good approximation, to Prot. This will not necessarily be the most healthy condition for forest-habitability of a planet orbiting a late $\mathrm{K}$ star or red dwarf, however, as the reduced photosynthetically active radiation (PAR) in red dwarf sunlight will be distributed over the whole surface of the planet as it rotates with respect to its sun. In the synchronous situation, perpetual daylight will compensate wholly, or in part, for the lower PAR fraction in insolation.

For $P_{\text {rot }} / P_{\text {orb }}=9 / 10$, night will last for 290.7 days for the M0 star and 189.7 days for the M2 star. Given that Earth's Cretaceous polar forests survived an annual cycle with half a year of darkness, the latter figure is not necessarily problematic, and the former might be entertained by postulating adjustments 
in plants' carbon budgets. As night grow longer, over geological time, it is not unreasonable to expect plants to adapt through evolutionary changes. This phase poses no ultimate threat to forest-habitability, but, at some point, there must come problems from the progressively extending nights. Note that when the ratio of $P_{\text {rot }} / P_{\text {orb }}$ becomes $99 / 100$, the day-night cycle lasts for 99 orbits, and night will last for 3,197 days, that is, 8.75 Earth years, on the planet of the M0 star. During this stage, a serious problem for trees will be the limited time available to make growth before the weakening light of the sinking sun, and the cooling air temperature compromised photosynthetic production. Long periods of dormancy would enable prolonged attack from organisms like fungi. Wolfe \& Upchurch (1986) and Wolfe (1991) investigated the likely damage to plant life that would have followed a prolonged episode of reduced photosynthetic production following the injection into the atmosphere of vast quantities of dust during the $\mathrm{K} / \mathrm{T}$ boundary impact. The spin-down of a planet to synchronous lock would entail repeated episodes of prolonged darkness, each one longer than that which preceded it. Unless a special continental configuration is postulated, whereby plants can migrate all the way around a planet, keeping up with the daylight, it is hard to avoid the conclusion that the survival of higher land plants would be very difficult.

Provided that the combination of tidal torque from the parent star and a planet's rate of tidal dissipation favoured a lengthy spin-down, there would come a time when trees (assuming that they had survived this far!) could reach reproductive maturity during the day, die off as they were carried closer to the terminator, and the species could survive as a seed bank overnight. Before a tree can set seed, it must first reach reproductive maturity, This can be a long process. Species surveyed in Zasada et al. (1992) are known to flower sporadically at an age of 15 years or less (Fowells 1965; Heinselman 1981). However, the age at which consistent production of seeds is possible under good growing conditions is generally 25 to 50 years (Koski \& Tallquist 1978). How long can seeds survive in the seed bank? Richards (1996) considered that large numbers of seeds might survive in tropical forest floor material for some years. Holthuijzen \& Boerboom (1982) demonstrated that seeds of Cecropia could remain viable in earthenware pots sunken into the ground for around 5 years 2 months (although survival under natural conditions might be more difficult). Zasada et al. (1992) summarised data on seed banks in boreal forests. Some seeds, such as those of Populus, are very short-lived and must germinate within days (Schreiner 1974). Seeds of other species do not germinate during the second growing season after they arrive on the forest floor. Fraser (1976) noted that seeds of Picea mariana remained viable on the forest floor for at least 10 months, but completely lost viability after 16 months. On the other hand, Granström (1987) found that the viability of Betula seeds in blocks of forest floor material artificially inserted into undisturbed forest floor, dropped from 100 to $60 \%$ over a five year period, although the seeds were probably not viable for more than 5 to 6 years in the seed bank. Granström and Fries (1985) found that the viability of seeds from Betula papyrifera fell from 100 to $6 \%$ in first year, but $3 \%$ of seeds remained viable after 3 years. Black spruce is of particular interest, because large quantities of seeds can be retained in semi-serotinous cones for up to 25 years - Haavisto (1975). 
Annuals do not offer us much more encouragement. Some seeds of desert annuals may remain viable for up to 10 years under artificial conditions (Went 1969), but Winkworth (1971) reported that seeds of the Australian buffel grass Cenchrus ciliaris lasted for a mere 2 years in the desert. Another stratagem to delay seed germination used by Blepharis persica, where mature fruit can remain protected by spiny bracts of the withered plant for several years (Gutterman 1972; but also see discussion in Archibold 1995). Having said that, the above generalisations do not exhaust the potential longevity of seeds and we cannot rule out the possibility of a long-lived seed bank.

Exactly how long "killer" nights would become before one side of the planet never emerged again into daylight depends on the precise circumstances, but in some cases (likely most common in the late $K$ to early $M$ dwarf stars) and certain initial planetary rotations, they might become very long indeed. An approximation for the time, $t$, for tidal locking of the rotation of the planet (after Whitmire \& Reynolds 1996) can be given as:

$$
t=\frac{Q}{P_{0} M^{2}}\left(\frac{r_{i}}{0.027}\right)^{6}
$$

where $Q$ is a friction parameter (usually taken as 13 for a planet with shallow seas, or 100 for a close to frictionless planet), $P_{0}$ is the initial rotation period of the planet, $M$ is the mass of the parent star, and $r_{i}$ is the planet's orbital semimajor axis (all in cgs units). A planet with a value of 13 , within the circumstellar habitable zone (i.e. receiving terrestrial insolation) of a $\mathrm{K} 5 \mathrm{dwarf}$ star, with an initial planetary rotation period of 10 and 20 hours would become tidally locked in 10.8 and 5.4 Gigayears, respectively. This same circumstance around an M5 star would occur in 14 and 7 million years, respectively. Thus, late M-stars may be expected to have a rate of tidal slowdown that might quickly bypass an extended period where are nearly equal, but studies must be done to isolate detailed instances specifically.

In any case, for slow rotation, global climate would have been moving ever closer to that on a SRP. This would mean that in effect, planetary rotation would carry vegetation through a series of day hemisphere climatic zones, before their locale reached the snow zone near the terminator. Marine algae would be in a better situation to survive, and one could stretch a point and ask whether sea-borne seeds such as coconuts would be a viable means of tree dispersal.

The safest conclusion appears to be that spin-down of NSRP into synchronous lock would involve closure of the forest-habitability window, if forests existed previously, but that this might re-open in suitable day hemisphere climatic regimes, once the planet had become a SRP. Higher land plants might then re-evolve from photosynthetic marine organisms.

\section{References}

Archibold, O. W. 1995, Ecology of World Vegetation (London: Chapman \& Hall)

Fowells, H. A. 1965, in Silvics of Forest Trees of the United States, Agricultural Handbook (Washington, D.C.:USDA Forest Service), No. 271, 
Fraser, J. W. 1976, Forestry Chronicle, 52, 229

Granström, A. 1987, Journal of Ecology, 75, 321

Granström, A. \& Fries, C. 1985, Canadian Journal of Forest Research, 15, 1176.

Gutterman, Y. 1972, Oecologia, 10, 145

Haavisto, V. F. 1975, in Black spruce symposium, Symposium Proceedings 0-P4, (Sault Saint Marie, Ontario: Canadian Forestry Service), 250.

Haberle, R., McKay, C. P., Tyler, D., \& Reynolds, R. 1996, in Circumstellar Habitable Zones: Proceedings of the First International Conference, ed. L. R. Doyle (Menlo Park, CA: Travis House Publications), 29

Heath, M. J. 1996, in Circumstellar Habitable Zones: Proceedings of the First International Conference, ed. L. R. Doyle (Menlo Park, CA: Travis House Publicationse), 445

Heath, M. J., Doyle, L. R., Joshi, M. M., \& Haberle, R. M. 1999, in Origins of Life, 29, 405

Heinselman, M. L. 1981, in Forest Succession: Concepts and Application, ed. D. C. West, H. H. Shugart, \& D. B. Botkin (New York, N.Y.: SpringerVerlag), 374

Holthuijzen, A. M. A. \& Boerboom, J. H. A. 1982, Biotropica, 14, 62

Joshi, M. M. 2004, this volume.

Joshi, M. M., Haberle, R. \& Reynolds, R. 1997, Icarus, 129, 450

Kasting, J. F., D. P. Whitmire, \& R.T. Reynolds 1993, Icarus, 101, 108.

Koski, V \& Tallquist, R. 1978, Forestali, 364, 1

Richards, P. W. 1996, The tropical rain forest: an ecological study, (Cambridge, U.K: Cambridge University Press, 2nd Edition)

Schreiner, E. J. 1974, in Seeds of woody plants in the United States, ed. C. S. Schopmeyer (USDA Forest Service Agricultural Handbook), 645 .

Went,F. W. 1969, Aliso, 7, 1

Whitmire, D.P. \& Reynolds, R.T., 1996, in Circumstellar Habitable Zones: Proceedings of the First International Conference, ed. L. R. Doyle (Menlo Park, CA: Travis House Publicationse)

Winkworth, R. E. 1971, Journal of Range Management, 24, 141

Wolfe, J. A. 1991, Nature, 352, 420

Wolfe, J. A. \& Upchurch, G. R. 1986, Nature, 324, 148

Zasada, J. C., Sharik, T. L. \& Nygren, M. 1992, in A Systems Analysis of the Global Boreal Forest, H. H. Shugart, R. Leemans \& G. B. Bonan (Cambridge, U.K.: Cambridge University Press), 85 


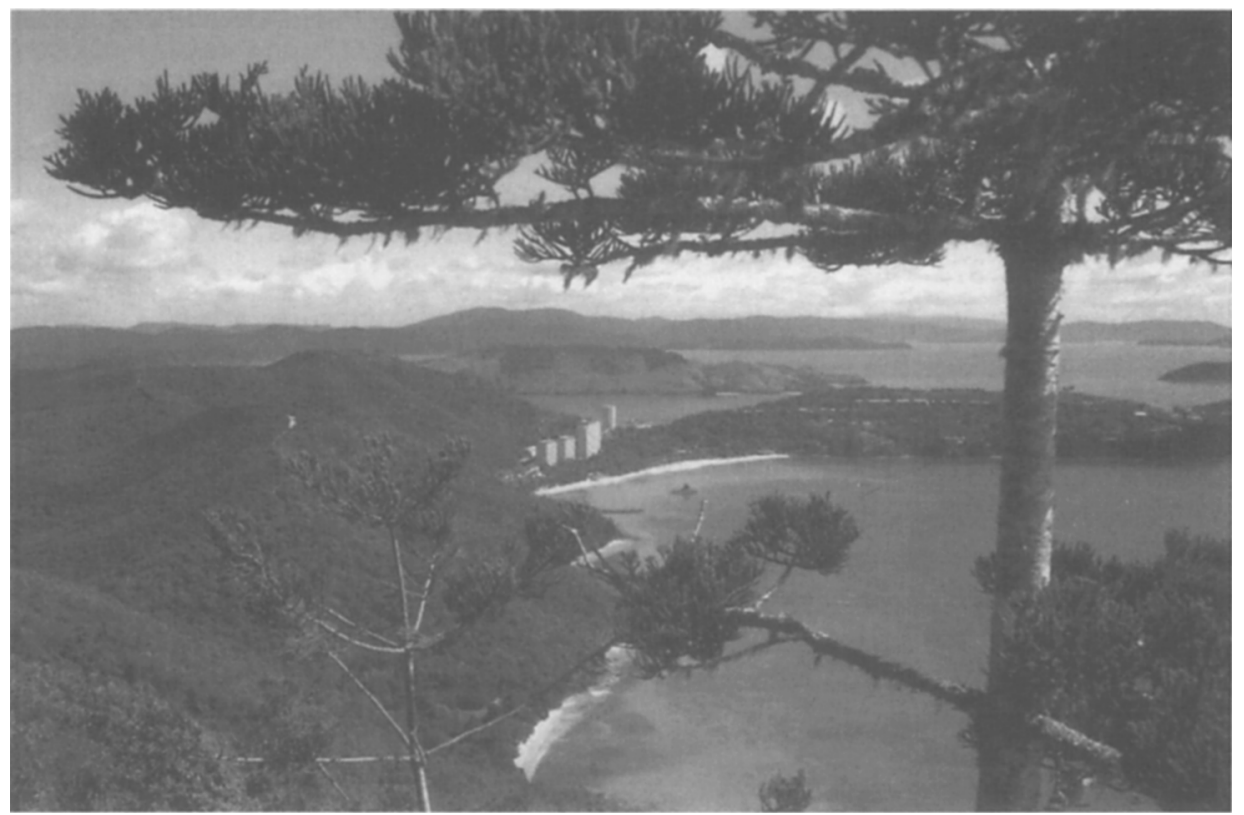

View looking back to conference centre hotel (photo: Seth Shostak) 